\title{
Efficient Multiphysics Finite Element Simulation of a Transient Fault in a Bi-2223 HTS Power Cable
}

\author{
Alexander N. Petrov, Member, IEEE, Igor O. Golosnoy, Senior Member, IEEE, and James A. Pilgrim, Senior \\ Member, IEEE
}

\begin{abstract}
In this work, the problem of a high temperature superconducting cable (HTSC) response to and recovery from a transient short circuit fault is discussed and a finite element solution to the problem is presented. The full model consists of three subroutines, connecting together 1) electromagnetic phenomena in HTSCs and associated Joule heat release, 2) heat transfer from the HTSC to the cooling circuit, 3) convective heat transport in liquid nitrogen. These subroutines are connected via geometric and variable couplings. The background of the real cable to be modelled is laid out together with its key properties. The most important parameter and variable calculations are explained in detail. In order to facilitate a computational speedup through manipulation of the active physics during selected intervals of time, the simulation is split in three distinct stages - steady-state, transient fault, and recovery. Moreover, it was shown how the entire simulation may be performed over a moderately reasonable amount of time using low computational resources when certain approximations are exploited - namely, using the electromagnetic model only on a limited number of cross-sections along the cable. This work shows that fairly accurate results can be achieved on an office PC using a commercial FEM software even without access to computing clusters, and that the same model can be used to examine both normal and transient operation.
\end{abstract}

Index Terms-Bi-2223, fault response, finite element, HTS modelling, power cable

\section{INTRODUCTION}

$\mathbf{I}$ $\mathrm{N}$ a standard power system, if a through fault is experienced by a high temperature superconducting (HTS) cable, the fault current would generate heat that could be enough to push the superconducting material into its normal resistive state. This in turn would generate even more heat. After the fault is cleared, a conventional cable would be almost immediately ready to conduct electricity again. A superconducting cable, however, needs some time for the coolant to bring the temperature back into working range. How long this time is depends highly on individual cable designs, as well as the magnitude and duration of the fault current.

There is a need for multiphysics models that can simulate both steady-state and transient conditions within a reasonable amount of time [1]. Such efficient models may be useful for the commercial implementation of superconducting cables as they will facilitate the theoretical design and understanding of how a particular design will behave in all possible situations.

Alexander N. Petrov and James A. Pilgrim were with the School of Electronics and Computer Science, University of Southampton, United Kingdom e-mail: anp2u12@soton.ac.uk

Igor O. Golosnoy is with the School of Electronics and Computer Science, University of Southampton, United Kingdom

Manuscript received February 15, 2021
However, simulating an HTS cable system via finite elements is usually computationally expensive. To decrease the computational time of a finite element model, certain assumptions or approximations are usually necessary and they are almost always expected to have decreased accuracy. In modelling of superconducting systems, such assumptions can often be brought down to two categories - simplification of geometry, or physics equations - both of which decrease the degrees of freedom, but usually at the cost of some accuracy. When used together, common simplifications include decreasing the spatial dimensions of the model from 3D to 2D, and often to 1D. While this offers a large speed-up, it also risks a large inaccuracy. This problem is amplified in systems such as superconducting cables, where a certain part of the cable must be modelled along its entire length (longitudinal temperature flow in the cable coolant) but another part simply cannot be (electromagnetic phenomena in the cable in 3D for the entire length of a cable are practically impossible and 1D would be very inaccurate). Therefore the preferred means of simulation have been via analytical or other, simpler, numerical methods [2]-[17], for example the equivalent circuit model. While these are fast and often reliable, they are not capable of describing the multiphysics at every point within the cable.

The contribution of this work is the construction and validation of a mulitiphysics FEM model for Bi-2223 HTS superconducting cables. The model bridges the gap between simulating the entire longitudinal coolant flow along the cable and the electromagnetic phenomena and power loss calculations that are best simulated in the cable's cross-section. The model can also be applied to both normal and transient operation. While the use of a computing cluster can decrease the computational time, the model can be ran on an office PC with 16 GB RAM, and it produced fairly accurate results in less than 60 hours. Further details are given in previous publications [18], [19].

A major contributor to the speed-up is the separation of each simulation step and each model subroutine. The subroutines are:

1. Longitudinal 1D thermal modelling of the cable coolant;

2. 2D electro-magneto-thermal modelling of the cable crosssection;

3. 2D-axisymmetric thermal modelling of the cable along its length.

The subroutines are coupled within the model. This provides a framework, on top of which the problem may be effectively solved by introducing the following simulation steps:

1. Steady-state temperature profile; 
2. Through fault generation (overcurrent response);

3. Post-fault recovery.

\section{MODEL FRAMEWORK}

This section describes the subroutines required to model a Triax superconducting cable with a two-channel coolant flow - inner supply and outer return.

\section{A. Modelling of the coolant}

Overview: The temperature flow in the coolant is examined using heat transfer in fluids physics applied on a 1D geometry. A 1D domain (a line) with length $l$ is built in the modelling environment. This domain represents the channel where the cooling liquid flows. A representation of this can be seen on Fig. 1 .

A point is defined at each $l_{\mathrm{dz}} \mathrm{m}$ along the length - this means a $u=l / l_{\mathrm{dz}}$ number of cross-sections will be modelled. As a 3D simulation of long HTS cables is in practice impossible, this paper attempts to observe what effect simulating a finite number of cross-sections will have on the accuracy of the simulation; the modelling of the cross-section is described later on. The points formed at each $l_{\mathrm{dz}}$ metres will also facilitate the coupling of the various subroutines.

In fluids, heat transfer generally occurs via conduction and convection. Particularly in cooling systems, heat is moved by forced convection that depends on the velocity of the fluid and other factors. The COMSOL module Heat Transfer in Fluids provides the $1 \mathrm{D}$ equation:

$$
\rho_{\mathrm{v}} C_{\mathrm{p}} \frac{\partial T}{\partial t}+v \rho_{\mathrm{v}} C_{\mathrm{p}} \frac{\partial T}{\partial z}=k \frac{\partial^{2} T}{\partial z^{2}}-q
$$

where $v$ - the velocity of the fluid in the pipe or channel, $\rho_{\mathrm{v}}$ - mass density, $C_{\mathrm{p}}$ - specific heat capacity, $T$ - temperature, $k$ - thermal conductivity (isotropic), $q$ - heat energy intake from neighbouring domains.

When there's an inner and outer coolant channels, two heat transfer physics are required. $T_{\mathrm{s}}$ is defined as the temperature of the supply flow and $T_{\mathrm{r}}$ is defined as the temperature of the return flow.

Boundary conditions: Each variable requires two boundary conditions. At $z=0 \mathrm{~m}, T_{\mathrm{s}}$ is equal to the inlet temperature of the coolant, whereas for $T_{\mathrm{r}}$ an outflow boundary condition is used. At $z=l \mathrm{~m}, T_{\mathrm{S}}$ needs the outflow boundary condition, whereas $T_{r}(l)=T_{\mathrm{S}}(l)$ - i.e. the outflow temperature of the supply flow is fed back into the return flow.

The inflow of heat from outside the cryostat wall into the return flow is implemented on the inner wall of the cryostat by specifying it as the general inward heat flux in $\mathrm{W} / \mathrm{m}^{2}$.

The heat transfer between the cable and the coolant is dependent on the heat transfer coefficient $h$, with the following notation used: $h_{\mathrm{s}}$ for the supply flow and $h_{\mathrm{r}}$ for the return flow. The heat flux crossing from the cable's wall to the coolant is, in general, described by:

$$
q=\operatorname{Ph}\left(T_{\text {wall }}-T_{\mathrm{ln}}\right)
$$

where $P$ - the perimeter of the surface where heat transfer occurs, $T_{\text {wall }}$ - the temperature of the cable's solid part at the boundary with the flow and $T_{\mathrm{ln}}$ - temperature of the coolant at the same location.

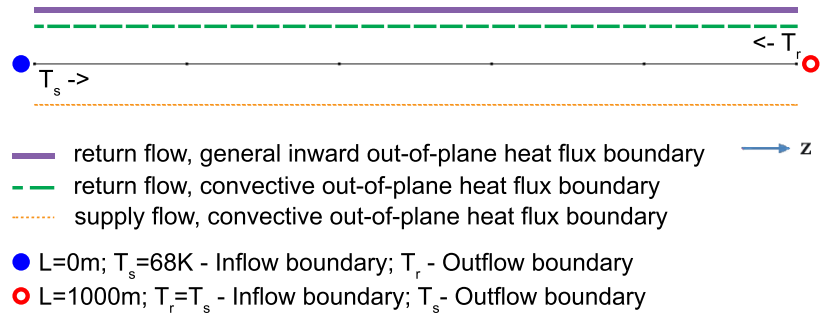

Fig. 1: The geometry and boundary conditions of the physics in the 1D coolant subroutine.

Heat transfer coefficients: The heat transfer coefficients $h_{\mathrm{s}}$ and $h_{\mathrm{r}}$ must be calculated accurately depending on the system properties. A detailed procedure for doing so is described in [20]. Firstly, the average fluid velocity $(\mathrm{m} / \mathrm{s})$ in a channel is described by:

$$
v=\frac{m}{A \rho_{\mathrm{v}, \mathrm{ln}}}
$$

where $m$ - mass flow (constant), $A$ - area of the channel (supply or return), $\rho_{\mathrm{v}, \mathrm{ln}}-$ mass density of liquid nitrogen. Then, the Reynolds number is described by:

$$
R e=\frac{\rho_{\mathrm{v}, \ln } v d}{\nu_{\mathrm{ln}}}
$$

where $d$ - internal diameter of the channel [21], $\nu_{\text {ln }}$ - liquid nitrogen viscosity. For the model in this paper, the Reynolds numbers for the supply and return flows are both larger than the transition value of 4000 (for pipes), therefore the flow in both supply and return flow is turbulent [21].

The formula for the heat transfer coefficient in turbulent flow is [22]:

$$
h=\frac{k_{\ln } 0.023}{d} \operatorname{Pr}^{0.4} \operatorname{Re}^{0.8}
$$

Where Pr is Prandtl's number:

$$
\operatorname{Pr}=\frac{\nu_{\mathrm{ln}} C_{\mathrm{p}-\mathrm{ln}}}{k_{\mathrm{ln}}}
$$

and $C_{\mathrm{p}-\mathrm{ln}}-$ specific heat capacity and $k_{\ln }-$ thermal conductivity of the liquid nitrogen.

As the steel former is in fact a corrugated tube, it is needed to modify the heat transfer coefficient to take this into account. According to [23], the new value for the supply flow heat transfer coefficient is:

$$
h_{s}^{*}=h_{s} \frac{F_{s}}{\pi\left(2 r_{s}-s_{s}\right)}
$$

where $F_{s}$ is the surface factor of the corrugated tube and $s_{s}$ is the wall thickness (Table III).

The final equations are:

$$
\begin{gathered}
h_{r}=\frac{k_{\ln } 0.023}{d_{r}}\left(\frac{\nu_{\ln } C_{\mathrm{p}-\ln }}{k_{\ln }}\right)^{0.4}\left(\frac{\rho_{\mathrm{v}, \ln } v_{r} d_{r}}{\nu_{\ln }}\right)^{0.8} \\
h_{s}^{*}=\frac{k_{\ln } 0.023}{d_{s}}\left(\frac{\nu_{\ln } C_{\mathrm{p}-\ln }}{k_{\ln }}\right)^{0.4}\left(\frac{\rho_{\mathrm{v}, \ln } v_{s} d_{s}}{\nu_{\ln }}\right)^{0.8} \frac{F_{s}}{\pi\left(2 r_{s}-s_{s}\right)}
\end{gathered}
$$




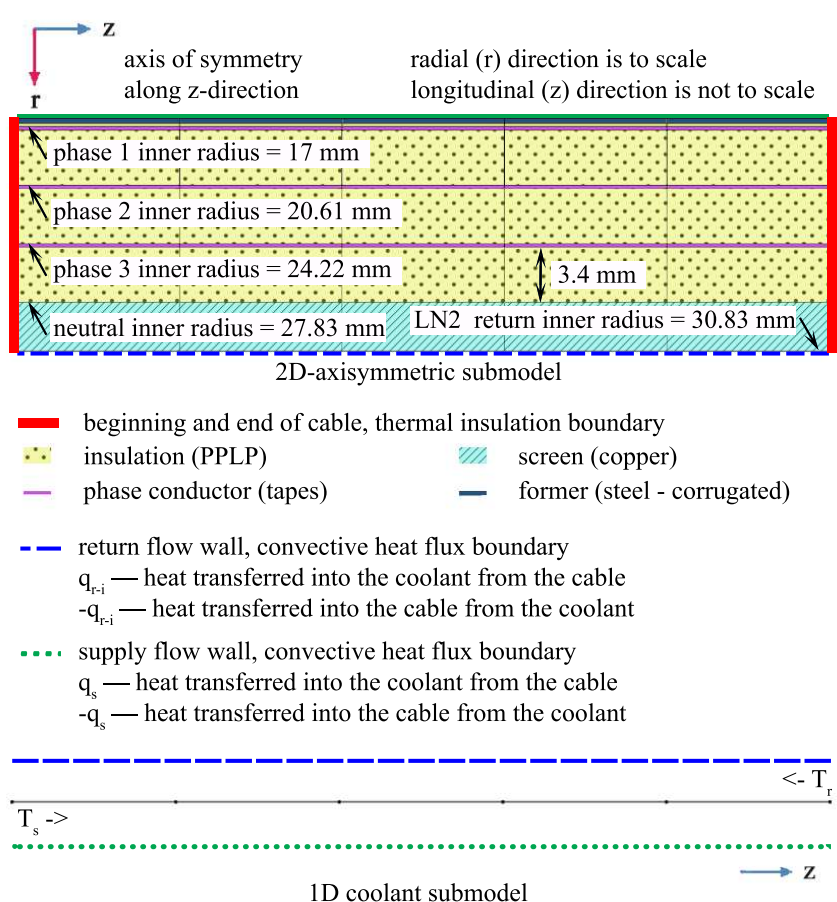

Fig. 2: The geometry and boundary conditions of the physics in the 2D-axisymmetric subroutine and its coupling with the 1D coolant subroutine.

\section{B. Modelling of the temperature along the total width and length of the cable}

Overview: Since a cable is often cylindrical and its construction consists of concentric rings - such as conductor, insulator - it may be assumed to be longitudinally symmetrical around its central axis. This results in a geometry with length $l$ that is many times larger than its width, however with adequate discretization, heat transfer physics may be modelled successfully.

The geometry of the 2D-axisymmetric subroutine consists of the former, insulation and conduction layers, as well as the neutral conductor. They are built for the entire length $l \mathrm{~m}$ of the cable. The geometry is divided via lines into $u$ equal parts with length $l_{\mathrm{dz}}-$ as explained in the previous section, this is to examine how well simulating several cross-sections can perform, and facilitates subroutine coupling.

Each phase is assumed to be a monoblock where the material is a mixture of HTS and silver. Hence, temperature rise in the 2D-axisymmetric model does not need any details of HTS geometry, just a uniform heat generation in the tape. The effective specific heat capacity and the effective mass density are linearly related to the fractions of HTS and silver material in the tape's cross-section. The thermal conductivity of the monoblock is assumed to be equal to the thermal conductivity of the HTS material, in line with literature [23].

Modelling of the temperature evolution in a superconductor is no different than any other material. Using the $2 \mathrm{D}$ equation for conductive heat transfer:

$$
\rho_{\mathrm{v}} C_{\mathrm{p}} \frac{\partial T}{\partial t}=k\left(\frac{\partial^{2} T}{\partial x^{2}}+\frac{\partial^{2} T}{\partial y^{2}}\right)+Q-q
$$

where $Q$ - heat energy from local heat source, and all other variables are as under (1).

Here, the temperature in the body of the cable is defined as $T_{0}$.

Boundary conditions: At the beginning $(z=0 \mathrm{~m})$ and the end of the cable $(z=l \mathrm{~m})$, along the radial direction (perpendicular to the axis of symmetry) the total heat transfer through the boundary is set equal to zero (thermal insulation). At the inner longitudinal boundary (parallel to the axis of symmetry), towards the supply flow, the convective heat transfer coefficient $h_{\mathrm{s}}^{*}$ is applied, see (9), and at the outer boundary, towards the return flow, the coefficient is $h_{\mathrm{r}}$, see (8). This setup is identical with the heat transfer in the 1D coolant physics, which ensures the conservation of heat once the models are coupled together.

Mesh and discretization: The mesh is divided, as in the 1D coolant physics, into 1000 elements along the length of the cable. A mapped mesh is used in order to minimize the size of the model. Due to the extremely high heat gradient between each phase and neighbouring domains, a boundary mesh layer must be introduced on the edges on each side of each phase. This helps combat the development of unrealistic temperature valleys.

In order to minimize the degrees of freedom, a linear discretization is used.

\section{Coupling of the coolant and cable body heat transfer physics}

So far three variables have been defined - the temperature of supply flow $T_{\mathrm{s}}$, the temperature of return flow $T_{\mathrm{r}}$ and the temperature within the body of the cable $T_{0}$. Within COMSOL, these variables use one physics module each for a total of three modules, and these are spread between two components (subroutines).

These variables do not share the same geometry, nor dimensions. In order to be able to simulate them together, it is necessary - regardless of the finite element modelling (FEM) software - to couple them.

As described in the previous subsection, the supply flow strictly exchanges heat with the inner wall of the cable itself ( $T_{s} \leftrightarrows T_{0}$ ), and the return flow strictly exchanges heat with the outer wall of the cable body ( $T_{r} \leftrightarrows T_{0}$ ), both while ensuring the conservation of energy holds. Both achieve this via a convective heat transfer boundary condition.

The heat transfer into the supply flow (and into the cable is the negative value) is:

$$
q_{s}=h_{\mathrm{s}}^{*}\left(T_{0(\text { wall,in })}-T_{\mathrm{s}}\right) P_{\mathrm{s}}
$$

And the heat transfer into the return flow (once again the heat into the cable will be the negative value) is:

$$
q_{\mathrm{r}-\mathrm{i}}=h_{r}\left(T_{0 \text { (wall,out })}-T_{\mathrm{r}}\right) P_{\mathrm{r}-\mathrm{i}}
$$

$T_{0 \text { (wall,in) }}$ and $T_{0 \text { (wall,out) }}$ can be seen as the green and blue boundary conditions in Fig. 2 , respectively - they are the same for the 2D-axisymmetric and 1D coolant models. 


\section{Modelling of the cable cross-section}

Only one quarter of the cable's cross-section is simulated, due to symmetry. Each individual tape in the cross-section is built using a homogenized superconducting domain, as described in detail in [18], and their widths are modified based on their phase as described in [19]. For the calculation of the Maxwell equations, the $H$-formulation is used. Symmetry in the model is implemented via Dirichlet boundary conditions on the $\mathrm{x}\left(H_{x}=0\right)$ and $\mathrm{y}\left(H_{y}=0\right)$ axes. The heat transfer equation of the 2D cross-sectional subroutine is allowed to run from the initial condition equal to the inlet temperature with the result of the 1D coolant steady-state simulation as constant boundary conditions until steady state is in turn reached for the temperature within the cross-section - this produces the initial conditions for the thermal part of the fault generation.

The $H$-formulation and the homogenization techniques used in this study have been verified in [18].

$H$-formulation: The $H$-formulation [24] has been successfully used to model current density and electromagnetic fields in superconducting tapes [25]-[27]. It has been used successfully in [18], [19].

Combining both Ampere's Law without a displacement field [28] and Faraday's Law with $J=E / \rho$ [29]:

$$
\nabla \times(\rho \nabla \times H)+\mu_{0} \mu_{\mathrm{r}} \frac{\partial H}{\partial t}=0
$$

where $H$ - magnetic field strength, $\rho$ - resistivity of the material, $\mu_{0}-$ magnetic permeability of free space, $\mu_{r}-$ relative permeability of the material, $\mathrm{J}$ - current density, E electric field. A well-rounded description of the $H$-formulation can be found in [28].

The initial conditions are:

$$
\begin{gathered}
H=\left[\begin{array}{l}
H_{x} \\
H_{y}
\end{array}\right]=\left[\begin{array}{l}
0 \\
0
\end{array}\right] \\
\frac{\partial H}{\partial t}=\left[\begin{array}{l}
0 \\
0
\end{array}\right]
\end{gathered}
$$

A Neumann boundary condition on the outer boundaries is set as per [25]:

$$
\frac{\partial H}{\partial t}=\left[\begin{array}{l}
0 \\
0
\end{array}\right]
$$

To introduce a given AC transport current, the current density on the entire conducting surface is integrated (in COMSOL, via a pointwise constraint) [27]:

$$
\int_{S} J_{\mathrm{n}} d S=I_{\mathrm{T}}
$$

where $S$ - surface on which the current is applied to the model, $J_{\mathrm{n}}$ - normal component of the current density on this surface (in 2D, the off-plane z-direction), $I_{\mathrm{T}}$ - total transport current.

Total power losses, in 2D, $Q(\mathrm{~J} / \mathrm{m})$ [30] are integrated [31], [32] over the duration of the fault $x$ and over the entire surface of the tape $S_{\mathrm{t}}$ :

$$
Q=\int_{0}^{x} \int_{S_{\mathrm{t}}} J E d t
$$

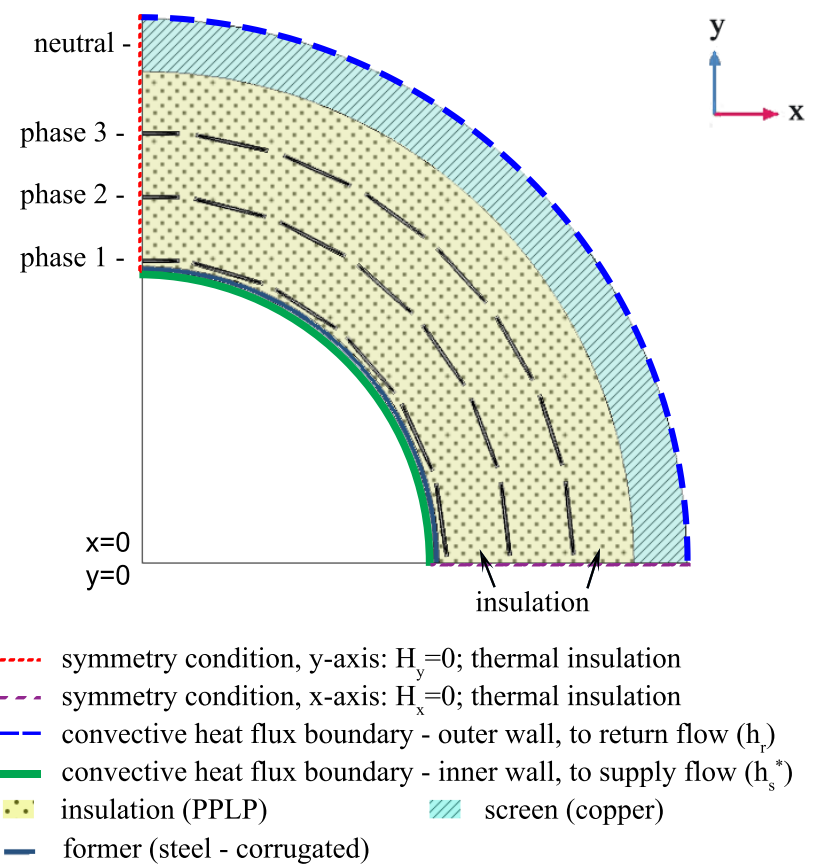

Fig. 3: The 2D cross-section for heat transfer, including boundary conditions. Note: the boundary conditions are different for the $H$-formulation.

Heat transfer: The simulated domains are only the three phases, the insulation and the neutral conductor. Then, there are three boundary conditions - the thermal insulation along the symmetry boundaries, the convective heat flux towards the supply flow ( $\left.T_{\mathrm{S}} \leftrightarrows T\right)$ and the convective heat flux towards the return flow $\left(T_{\mathrm{r}} \leftrightarrows T\right)$. Heat generation within the conductors is achieved via the product of current density and electric field $J E$.

Regarding the convective heat flux boundaries, the heat transfer coefficients are $h_{\mathrm{S}}^{*}$ and $h_{\mathrm{r}}$ as calculated in the previous section. The external temperature at the boundaries is equal to the liquid nitrogen temperature of the supply $T_{\mathrm{s}}$ or return flow $T_{\mathrm{r}}$, which are taken at their corresponding points every $l_{\mathrm{dz}}$ metres along the length of the cable (more on that later). An illustration of all boundary conditions in one cross-section of the system is shown in Fig. 3 .

\section{E. Temperature distribution within the $2 D$-axisymmetric model}

To effectively analyze the temperature profiles in the coupled 1D and 2D-axisymmetric system, the results of the discrete simulation of each cross-section must be considered.

As already described, the 2D-axisymmetric subroutine is split in $u$ sectors, and each sector is bound by an upper (towards the end of the cable) and lower (towards the beginning of the cable) cross-section. The heat that would be generated in a given phase, at a given point between any two crosssections, at a given time in the $2 \mathrm{D}$-axisymmetric subroutine may be interpolated from same conditions in the 2D crosssection subroutine simulations for those two cross-sections. The heat generated in the $2 \mathrm{D}$ cross-section is the product 
of the local current density $J$ and the electric field $E$. The difference between the volumes of the monoblock in the 2Daxisymmetric model and the tapes in the 2D cross-section model are taken into account to ensure the amount of energy is the same in each model.

\section{F. Equivalent resistance, and temperature dependencies}

Let $A_{\mathrm{hts}}$ - area of the tape's actual superconducting material in the tape cross-section, $A_{\text {hom }}$ - area of the tape's homogenized superconducting domain as built in the model, $A_{\mathrm{Ag}}-$ area of the tape's actual matrix material (silver) in the tape cross section.

Critical current and resistivity: The local critical current density of the superconductor is [33]:

$$
J_{\mathrm{c}}(B, T)= \begin{cases}0, & \text { if } T \geq T_{\mathrm{c}} \\ J_{\mathrm{c} 0} \times f\left(B_{\perp}\right) g\left(B_{\|}\right) m(T), & \text { otherwise }\end{cases}
$$

Here $f\left(B_{\perp}\right)$ and $g\left(B_{\|}\right)$are the functions describing the critical current's magnetic field dependence. $m(T)$ is the critical current temperature dependence function normalized by $J_{\mathrm{c} 0}$ [34]:

$$
m(T)=\frac{T_{\mathrm{LN}_{2}}}{\left(1-T_{\mathrm{LN}_{2}} / T_{\mathrm{c}}\right)^{3 / 2}} \times\left(1-\frac{T}{T_{\mathrm{c}}}\right)^{3 / 2}
$$

where $T_{\mathrm{LN} 2}$ is the reference temperature (usually $77 \mathrm{~K}$ ).

If $\rho_{\text {plaw }}$ holds the power law:

$$
\rho_{\text {plaw }}=\frac{E_{\mathrm{c}}}{J_{\mathrm{c}}(B, T)}\left|\frac{J}{J_{\mathrm{c}}(B, T)}\right|^{n-1}
$$

where $J$ - the local current density, $B$ - local magnetic field, $E_{\mathrm{c}}-$ critical current criterion (electric field equal to $10^{-4} \mathrm{~V} / \mathrm{m}$ ). Then the resistivity of the actual superconducting material $\rho_{\text {hts }}$ is based on [35]:

$$
\rho_{\text {hts }}=\frac{\rho_{\text {plaw }} \rho_{\mathrm{n}}}{\rho_{\text {plaw }}+\rho_{\mathrm{n}}}
$$

where $\rho_{\mathrm{n}}(T)$ - the normal state resistivity of the superconducting material.

If $s_{\text {hom,ag }}$ is the fraction of silver in the domain:

$$
s_{\mathrm{hom}, \mathrm{ag}}=\frac{A_{\mathrm{hom}}-A_{\mathrm{hts}}}{A_{\mathrm{hom}}}
$$

Then:

$$
\rho_{\mathrm{hom}}=\frac{1}{\left(1-s_{\mathrm{hom}, \mathrm{ag}}\right) / \rho_{\mathrm{hts}}+s_{\mathrm{hom}, \mathrm{ag}} / \rho_{\mathrm{Ag}}}
$$

$\rho_{\mathrm{Ag}}(T)$ is the resistivity of the silver matrix material. $\rho_{\mathrm{hom}}$ is used within the model by the $H$-formulation to calculate the resistivity within the homogenized domain and $\rho_{\mathrm{hts}}$ is the "real" resistance of the superconducting material. This separation is needed because the homogenized domain approximation was used in the model to avoid modelling of fine details of HTS filament, and using circuit equations requires resistance values as they would be in reality.

Additionally, the temperature dependencies of the normal state resistivity $\rho_{\mathrm{n}}$ and silver resistivity $\rho_{\mathrm{Ag}}$ must be defined.

The values of specific heat and mass density in the homogenized HTS domain are linearly based on the fraction of HTS and silver material within it; the value of the thermal conductivity is the same as in the Bi-2223 material [23].
TABLE I: Geometric specification of the simulated cable. Information is extracted from [23], [36]-[38] and private communication (Dr Wescley Tiago Batista de Sousa, Karlsruhe Institute of Technology, October 2019).

* - PPLP stands for polypropylene laminated paper.

\begin{tabular}{ll}
\hline \# of phases (\# layers per phase) & $3(1)$ \\
\# tapes per layer (Ph1/Ph2/Ph3) & $22 / 26 / 30$ \\
Tape twist angle & $16^{\circ}$ (all phases) \\
Inner radius of 1st phase & $17 \mathrm{~mm}$ \\
Inner radius of 2nd phase & $20.61 \mathrm{~mm}$ \\
Inner radius of 3rd phase & $24.22 \mathrm{~mm}$ \\
Inner radius of copper neutral & $27.83 \mathrm{~mm}$ \\
Inner radius of $\mathrm{LN}_{2}$ return channel & $30.83 \mathrm{~mm}$ \\
Inner radius of cryostat & $40 \mathrm{~mm}$ \\
Thickness of PPLP* insulation layer & $3.4 \mathrm{~mm}$ \\
Cable length & $1000 \mathrm{~m}$ \\
\hline Tape dimensions & $4 \mathrm{~mm} \times 0.21 \mathrm{~mm}$ \\
Ratio of HTS to total area & 0.3 \\
Ratio of Ag to total area & 0.7 \\
\hline
\end{tabular}

Resistance of the cable: The average resistance per metre of the superconductor material of the cable $R_{\mathrm{hts}, k}$ at a given time can be found by obtaining $\rho_{\mathrm{hts}, \text { ave, } k}$ - the average value of the resistivity $\rho_{\text {hts }}$ within the homogenized superconducting domain. Then:

$$
R_{\mathrm{hts}, k}=\frac{\rho_{\mathrm{hts}, \text { ave }, k}}{n A_{\mathrm{hts}}}
$$

where $k=1 ; 2 ; 3$ indicates the phase and $n$ is the corresponding number of tapes per phase.

Similarly, for the matrix material:

$$
R_{\mathrm{Ag}, \mathrm{k}}=\frac{\rho_{\mathrm{Ag}, \mathrm{ave}, \mathrm{k}}}{n A_{\mathrm{Ag}}}
$$

where $\rho_{\mathrm{Ag} \text {,ave, } k}$ is the average value of the resistivity $\rho_{\mathrm{Ag}}$ within the silver matrix material. Then, the total resistance of a phase $k$ can be expressed as two resistances connected in parallel:

$$
R_{k}=l_{k} \frac{R_{\mathrm{hts}, k} R_{\mathrm{Ag}, k}}{R_{\mathrm{hts}, k}+R_{\mathrm{Ag}, k}}
$$

where $l_{k}$ is the actual length of the tapes within the cable. When the layers are twisted, $l_{k}$ is always a little larger than the total length of the cable:

$$
l_{k}=\frac{l}{p_{k}} \sqrt{p_{k}^{2}+c_{k}^{2}}
$$

where $p_{k}$ is the pitch length of the layer $k$ and $c_{k}$ is the layer circumference (where the radius is taken through the exact middle of the layer).

The resistance with which the transient fault is simulated $R_{\text {tot }, k}$ must also include the lumped resistance of the grid upstream from the HTS cable $R_{\text {grid }}$ :

$$
R_{\text {tot }, k}=R_{k}+R_{\text {grid }}
$$


TABLE II: Operational specification of the simulated cable. Information is obtained from [36] and private communication (Dr Wescley Tiago Batista de Sousa, Karlsruhe Institute of Technology, October 2019)

* - due to lack of information for the magnetic field dependence of the tape in this paper, it has been assumed to be similar to the field dependence of an earlier tape from literature.

\begin{tabular}{ll}
\hline Tape $I_{c}(77 K, 0 T)$ & $200 \mathrm{~A}$ \\
Tape $n(77 K, 0 T)$ & 14 \\
Tape $B$-dependence & From $[39]^{*}$ \\
Total $I_{\mathrm{c}}(\mathrm{Ph} 1 / \mathrm{Ph} 2 / \mathrm{Ph} 3)$ & $4400 \mathrm{~A} / 5200 \mathrm{~A} / 6000 \mathrm{~A}$ \\
RMS phase voltage & $10,000 \mathrm{~V}$ \\
3-phase fault duration & $0.1 \mathrm{~s}$ \\
$\mathrm{R}_{\text {grid }}$ & $0.04 \Omega$ \\
$\mathrm{L}_{\text {grid }}$ & $0.58 \Omega$ \\
$\mathrm{LN}_{2}$ intake temperature & $68 \mathrm{~K}$ \\
$\mathrm{LN}_{2}$ mass flow rate & $0.425 \mathrm{~kg} / \mathrm{s}$ \\
Cryostat heat leakage & $1.7 \mathrm{~W} / \mathrm{m}$ \\
Viscosity $\nu_{\mathrm{LN} 2}$ & $166.678 \times 10^{-6} \mathrm{~Pa} \cdot \mathrm{s}$ \\
\hline
\end{tabular}

TABLE III: Parameters of the former (corrugated tube) in the simulated cable. Information is extracted from [23]

\begin{tabular}{llr}
\hline Wall thickness & $0.3 \mathrm{~mm}$ & $\mathrm{~s}_{\mathrm{s}}$ \\
Surface factor & $0.146 \mathrm{~m}^{2} / \mathrm{m}$ & $\mathrm{F}_{\mathrm{s}}$ \\
Groove depth (tube width) & $1 \mathrm{~mm}$ & $t_{\mathrm{gr}}$ \\
\hline
\end{tabular}

\section{Simulation STEPS}

\section{A. The cable at hand}

In this subsection, a few of the parameters unique to the cable (and model) at hand are presented. All remaining parameters are presented in Tables II to IV

In the cable at hand, $l=1000 \mathrm{~m}$. The mesh for the $1 \mathrm{D}$ coolant domain and the 2D-axisymmetric domains is divided into 1-metre-long elements. $l_{\mathrm{dz}}=l / 5=200 \mathrm{~m}$ along the length - this means six cross-sections will be modelled; this number appears to be a reasonable compromise between simulation time and accuracy for the purposes of this investigation. The discretization is linear.

The geometry and all boundary conditions can be seen on Fig. 1. A compressed, not-to-scale representation of the final 2D-axisymmetric geometry can be seen in Fig. 2

The temperature dependencies of the normal state resistivity $\rho_{\mathrm{n}}$ and silver resistivity $\rho_{\mathrm{Ag}}$ are defined:

$$
\begin{gathered}
\rho_{\mathrm{n}}=4.53 \times 10^{-8} \times T_{\mathrm{hts}}+1.15 \times 10^{-6} \\
\rho_{\mathrm{Ag}}=7 \times 10^{-11} \times T_{\mathrm{Ag}}-2 \times 10^{-9}
\end{gathered}
$$

where $T_{\text {hts }}$ and $T_{\mathrm{ag}}$ are the temperatures of the HTS and silver material, respectively. All these values are graciously provided by Dr Wescley Tiago Batista de Sousa from KIT through private communication.
TABLE IV: Thermal parameters of the materials of the simulated cable. All values are obtained through private communication with Dr Wescley Tiago Batista de Sousa from KIT in relation to [36].

\begin{tabular}{lrrr}
\hline & $\mathrm{k}(\mathrm{W} / \mathrm{m} \cdot \mathrm{K})$ & $\mathrm{C}_{\mathrm{p}}(\mathrm{J} / \mathrm{kg} \cdot \mathrm{K})$ & $\rho_{\mathrm{v}}\left(\mathrm{kg} / \mathrm{m}^{3}\right)$ \\
Bi-2223 & 150 & 200 & 5600 \\
Silver & 480 & 225.2 & 10470 \\
St. Steel & 1 & 200 & 7900 \\
Copper & 534.186 & 244.6 & 8960 \\
PPLP & 0.147051 & 430 & 1098 \\
$\mathrm{LN}_{2}$ & 0.147051 & 2024.95 & 811.229 \\
\hline
\end{tabular}

\section{B. Simulation of the steady state}

A steady state temperature distribution of the coolant along the simulated cable was calculated in [36].

In the proposed algorithm, it is necessary to run the coupled 1D and 2D-axisymmetric subroutines described above in a time-dependent solver. A PARDISO or MUMPS direct solver with a Fully Coupled approach are found to be sufficient for the task and the relative tolerance can be set to 0.0001 [40]. The solver must simulate for a sufficiently long time to ensure the temperature has settled in a steady-state; for the model simulated in this paper it was observed that $10^{6}$ seconds is long enough for that to happen. Only the starting (0 seconds) and ending $\left(10^{6}\right.$ seconds) points in time need be saved.

The initial conditions are equal to the inlet temperature. The Ohmic losses during the steady state calculation are assumed negligible and so the change in the thermal state of the system is driven by the external heat flux coming through the cryostat.

\section{Solving for the duration of the fault}

The cable is split in $u$ equal parts of $l_{\mathrm{dz}} \mathrm{m}$ each. These are bounded by $u+1$ individual cross-sections, and each one of these is modelled separately. Such a split is required since the temperature rise along the cable may be non-uniform and thus may not be simulated via a single cross-section. In this paper, the choice of the number of these cross-sections is an educated guess. There are two ways to simulate these cross-sections.

1) Distributed parametric sweep, where each cross-section is decoupled from the rest and simulated in parallel. The difference between the values of the calculated damped transient fault current between each cross-section is assumed negligible.

2) Simultaneously running all cross-sections within one simulation. Here the current depends on the whole cable, which is more accurate, but at the cost of simulation time. Each cross-section requires its own unique set of variables which increases the model complexity.

Considering the variables described throughout the previous sections, this is the basic Kirchoff law equation for the system:

$$
V_{\mathrm{s}, k} \sin \left(\omega t+\phi_{k}\right)=R_{\mathrm{tot}, k} I_{k}+L \frac{d I_{k}}{d t}
$$

$V_{\mathrm{s}, k}$ - peak source voltage, line to neutral, $\omega$ - angular frequency of the source voltage, $\phi_{k}$ - the phase shift and $\phi_{k}=0^{\circ} ; 120^{\circ} ; 240^{\circ}$ for $k=1 ; 2 ; 3$ respectively. $I_{k}$ is the 


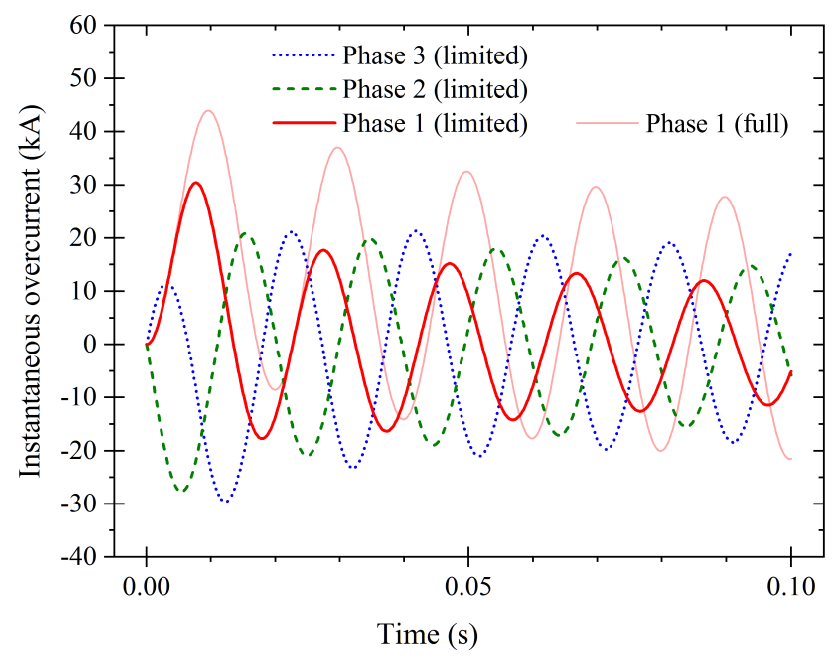

Fig. 4: The simulated full and limited fault currents in the cable model.

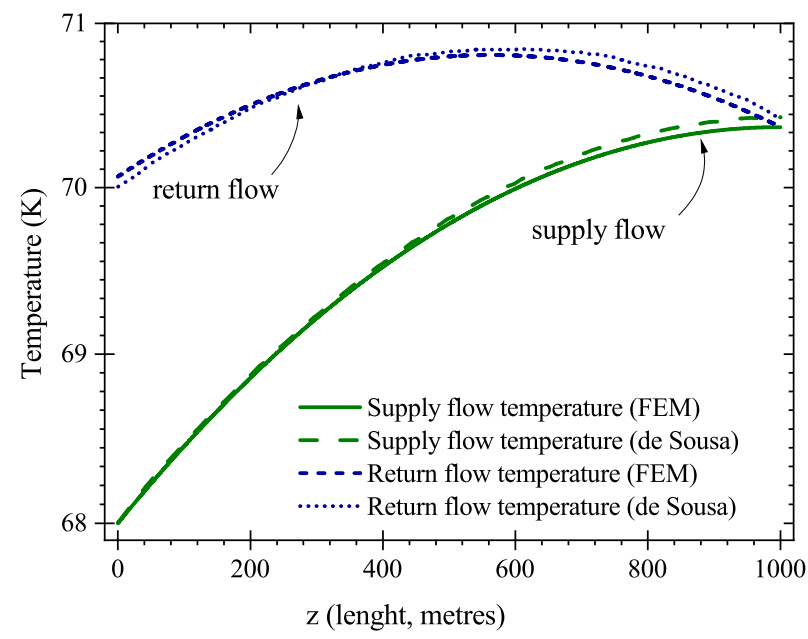

Fig. 5: The steady-state temperature along the simulated cable. The comparison is between the simulated values via FEM and the finite differences model in [36].

total current flowing in each phase, calculated by integration over all tapes of that phase (and accounting for symmetry if applicable). The constant inductance $L$ includes both the inductance upstream along the grid and the inductance of the cable.

If $R_{\text {tot }, k}=R_{\text {grid }}$, then the non-damped transient fault current can be obtained.

\section{Recovery from the fault}

After the fault has been cleared, the cable can begin recovery. The temperature of the superconducting material must drop to a value sufficiently below its critical value, whereas the temperature of the coolant must remain under its boiling value (78 $\mathrm{K}$ for liquid nitrogen).

The same physics are used as already described, with the exception of the initial conditions, which are set to begin the simulation from the end of the fault. Initially, the $\mathrm{H}$ -

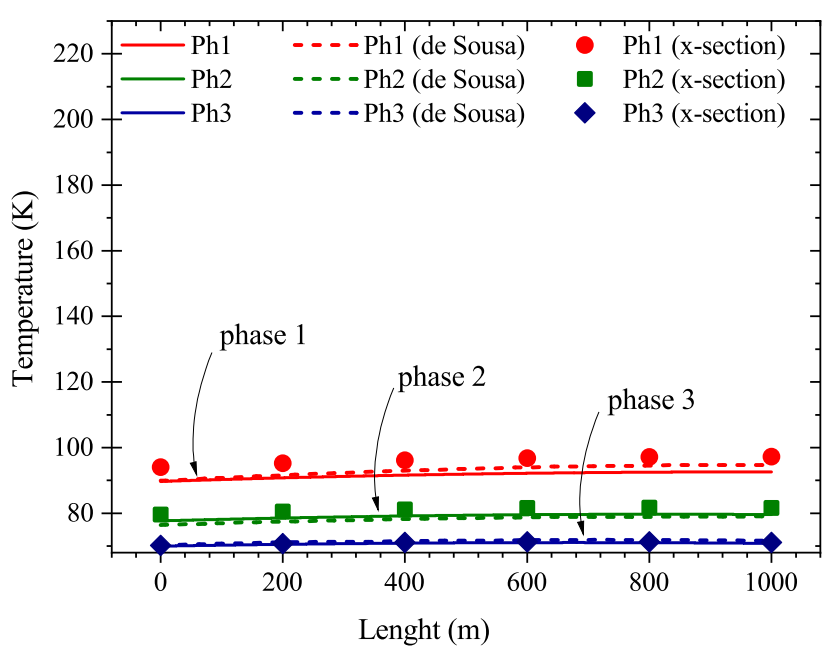

(a) $t=0.01 \mathrm{~s}$

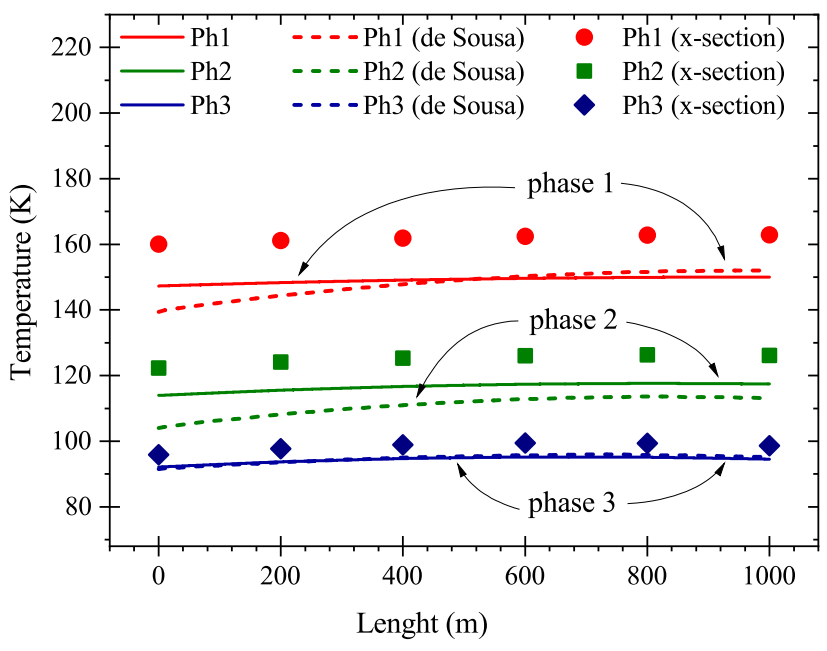

(b) $t=0.05 \mathrm{~s}$

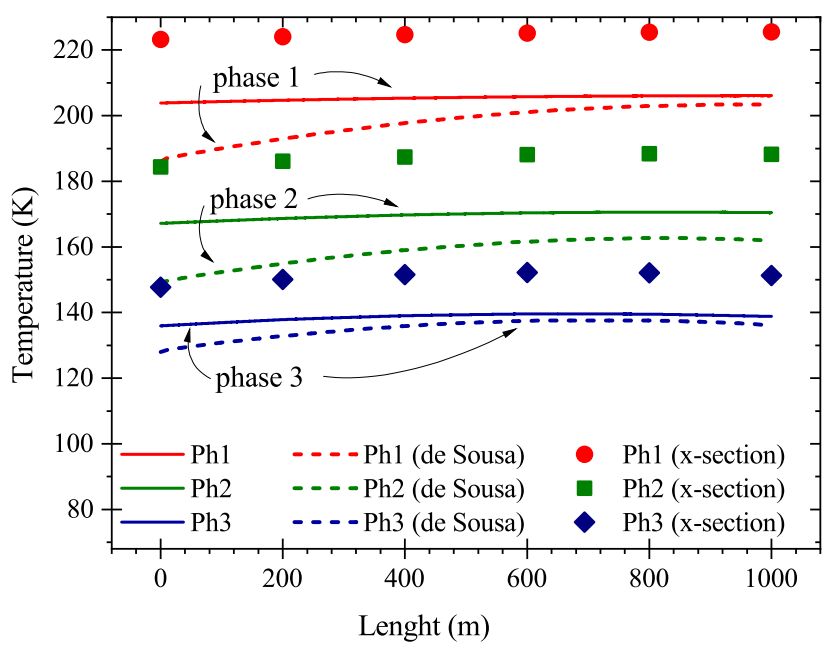

(c) $t=0.1 \mathrm{~s}$

Fig. 6: The temperature distribution in each phase along the cable length for three times during the fault, taken from the 2D-axisymmetric solution (solid lines) and the

2D-cross-section solution (crosses), compared with the published values from [36] (dashed lines). 


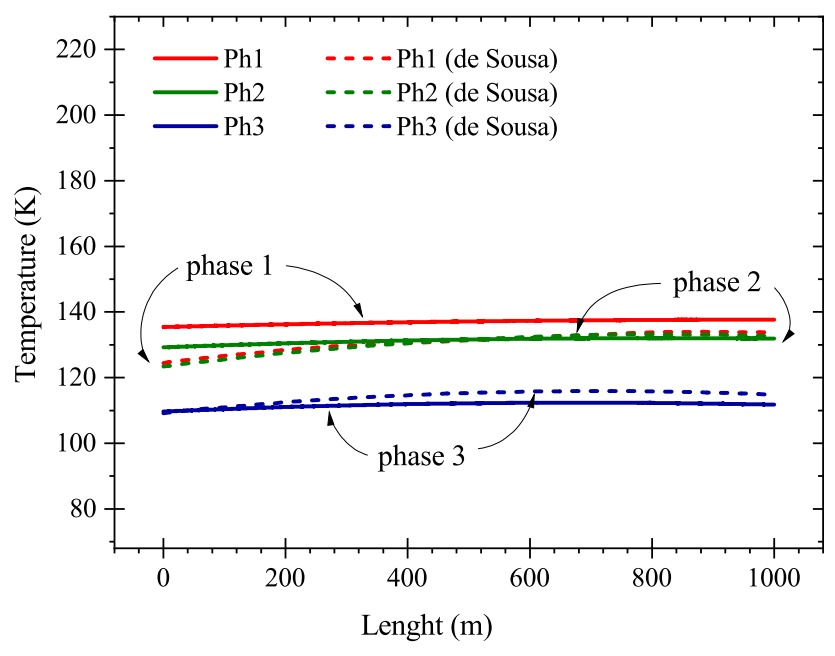

(a) $t=0.5 \mathrm{~s}$

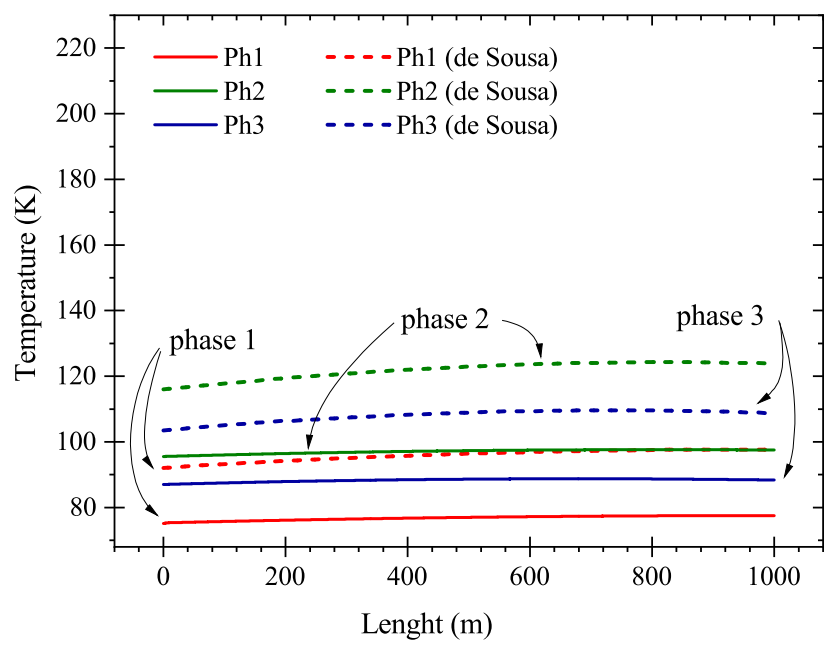

(c) $t=5 \mathrm{~s}$

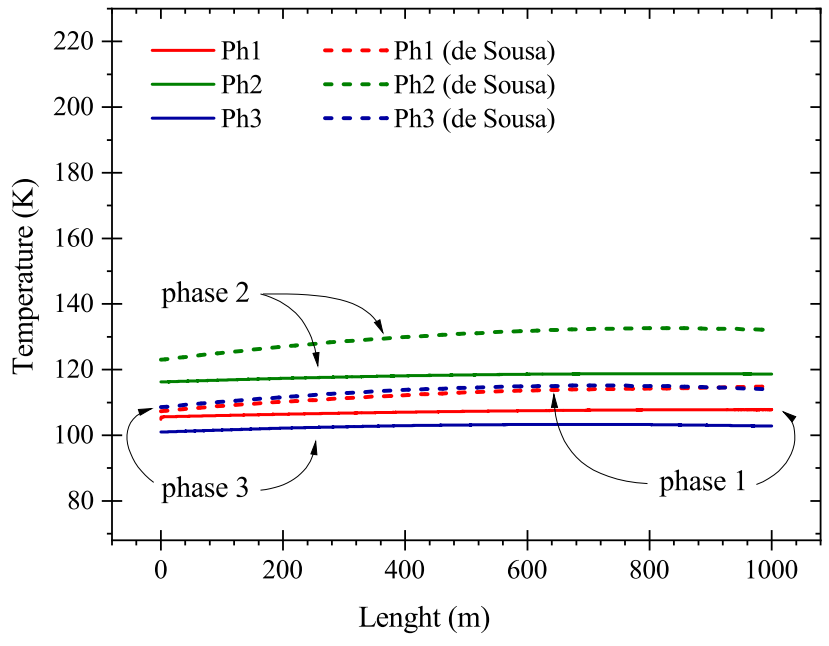

(b) $t=1 \mathrm{~s}$

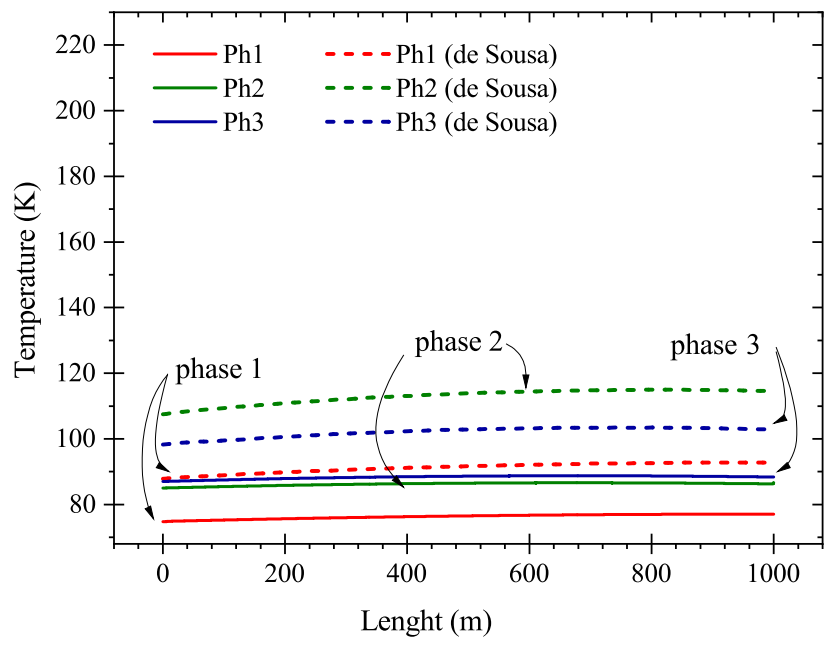

(d) $t=10 \mathrm{~s}$

Fig. 7: The recovery of the temperature, and its distribution, in each phase along the cable length for four times after the fault has been cleared, taken from the 2D-axisymmetric solution (solid lines) and compared with the published values from

[36] (dashed lines).

formulation is turned off and only heat transfer is allowed to run - further details can be found in the next section. The 1D heat transfer subroutine can be ran in conjunction with the 2Daxisymmetric subroutine, simultaneously simulating for the temperature of the coolant and the cable.

\section{STEAdy STATE AND FAUlt SIMULATION RESUltS}

\section{A. Steady state}

A simulation on a desktop PC with Intel i7-6700 CPU, 3.40 $\mathrm{GHz}$, with $16 \mathrm{~GB}$ of RAM, managed to solve for 735,000 degrees of freedom in 3 minutes and 18 seconds.

The result of the simulation for the cable at hand, together with the extracted data from [36], is available in Fig. 5] The same profile has been observed elsewhere in literature as well [41]. The temperature of the coolant at the outflow of the cable is $70.07 \mathrm{~K}$, marginally above the prescribed value of $70 \mathrm{~K}$; the maximum temperature is $70.8 \mathrm{~K}$ which is a little under the $70.85 \mathrm{~K}$ from the FD model in [36]. The length at which the maximum temperature occurs is 562 metres instead of 610-620 metres, and the temperature at the end of the cable is $70.37 \mathrm{~K}$ instead of $70.42 \mathrm{~K}$.

The inequalities may be attributed to the different model construction between finite differences and finite elements.

\section{B. Fault simulation}

In Fig. 4 the values of the simulated currents are presented for the entire duration of the three-phase fault. The values are very close to those obtained in [36], with the first peak of the limited current being calculated as $30,420 \mathrm{~V}$ and the value from literature equal to about $32,000 \mathrm{~V}$. This also shows the method of calculation of the resistance is reasonable.

The temperature distribution along each phase in the cable is shown on Fig. 6, for three instances in time while the fault is on-going. The solid lines represent the simulated temperatures in the 2D-axisymmetric model, the dashed - the values for the temperatures from literature, and the crosses - the highest 


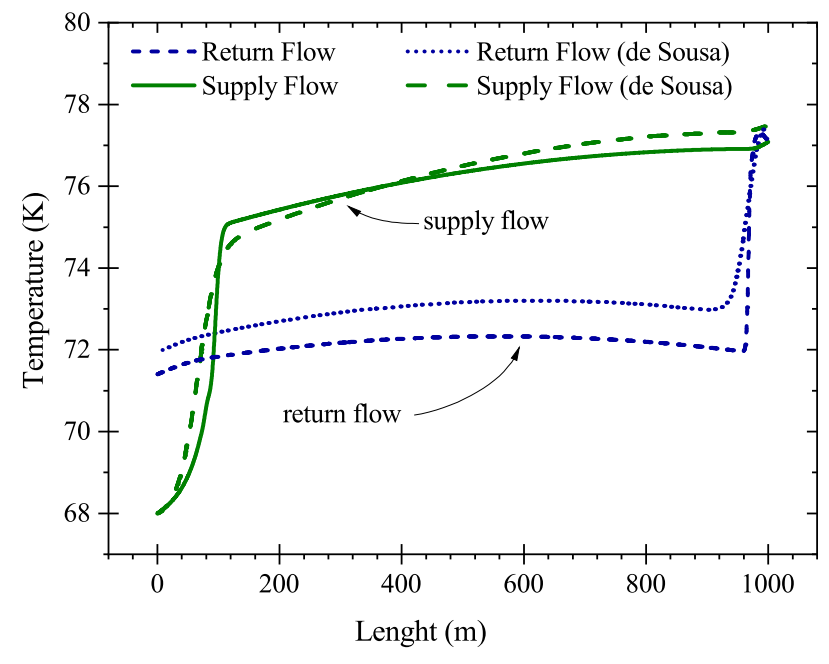

(a) $t=3 \mathrm{~min}$

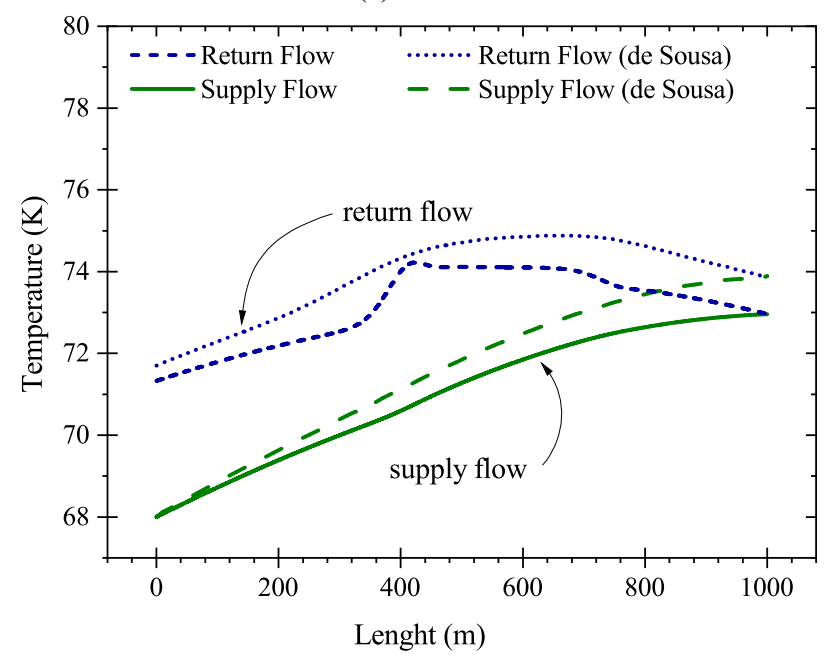

(c) $t=1 \mathrm{~h}$

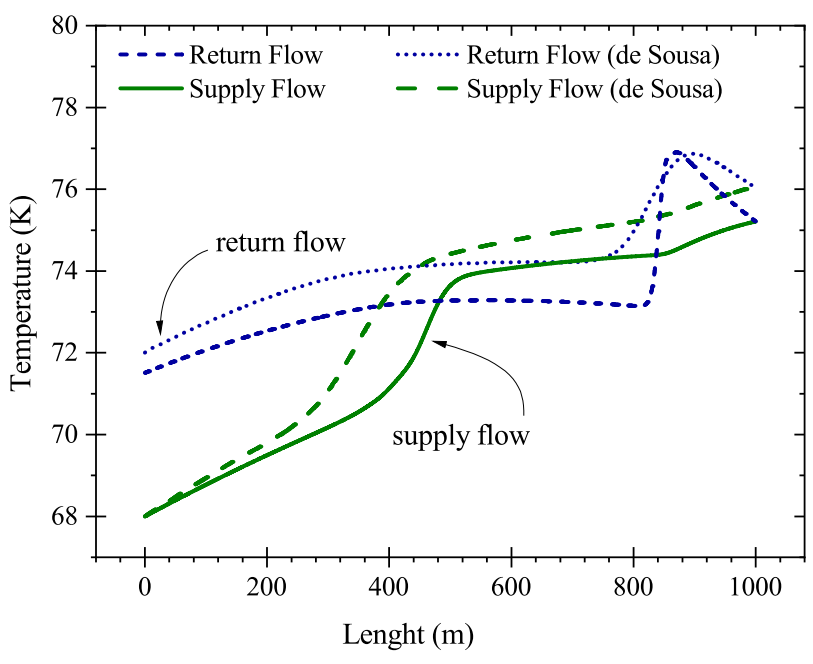

(b) $t=15 \mathrm{~min}$

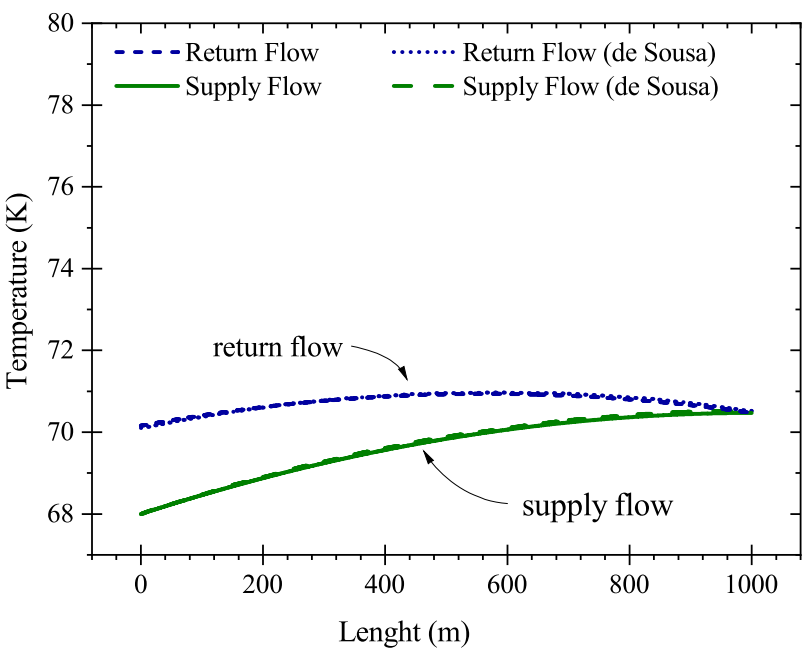

(d) $t=8 \mathrm{~h}$

Fig. 8: The recovery of the temperature, and its distribution, in the coolant along the cable length for four times after the fault has been cleared, taken from the 1D solution (solid lines) and compared with the published values from [36] (dashed lines).

value of the temperature in the $2 \mathrm{D}$ cross-section model at the corresponding length and phase. The generated losses for the duration of the fault for phase 1 are $8 \mathrm{~kJ} / \mathrm{m}$, for phase 2 are $7 \mathrm{~kJ} / \mathrm{m}$, for phase 3 are $5.6 \mathrm{~kJ} / \mathrm{m}$, and for the copper neutral are $15 \mathrm{~J} / \mathrm{m}$. The loss waveform follows a shape similar to the one observed in Fig. 4

The first phase sees the largest increase due to having the lowest critical current; this is also recognized in [36]. Another result coming from the lower critical current is that the first phase also experiences the strongest current limitation.

Phase two generates heat faster than the third phase and slower than the first. This holds true from the onset of the fault to its clearing.

The calculated temperature at the time of clearing of the fault is higher than the results presented in literature. The literature suggests a 10-25 $\mathrm{K}$ difference along the length of the cable for phase one, however the simulation shows only $5 \mathrm{~K}$. The result (in the distributed parametric solution) is not affected by the approximation that the transient current may be simulated within each cross-section without regard to the remaining parts of the cable, because running all cross-sections as one model (and thus equal transient current) yielded results with negligible difference. This shows that the initial temperature difference along the cable did not seem to affect the rate of temperature rise. A single cross-section may be more than sufficient to simulate fault current in the entire cable, however, this may not be the case for other cable designs or for simulating normal operation.

Otherwise, the difference between the calculated temperatures in the 2D-axisymmetric subroutine and the temperature from the literature at $t=0.1 \mathrm{~s}$ (Fig. 6), where the the temperature is highest is $1.5 \%, 4.9 \%, 1.4 \%$ for phases one through three respectively; and for the beginning of the cable is $8.9 \%$, $10.7 \%, 5.9 \%$.

Another notable result is the visibly higher temperature obtained from the 2D cross-section subroutine when compared to the axisymmetric subroutine. This expected, since the way the two subroutines handle the area of conducting material 


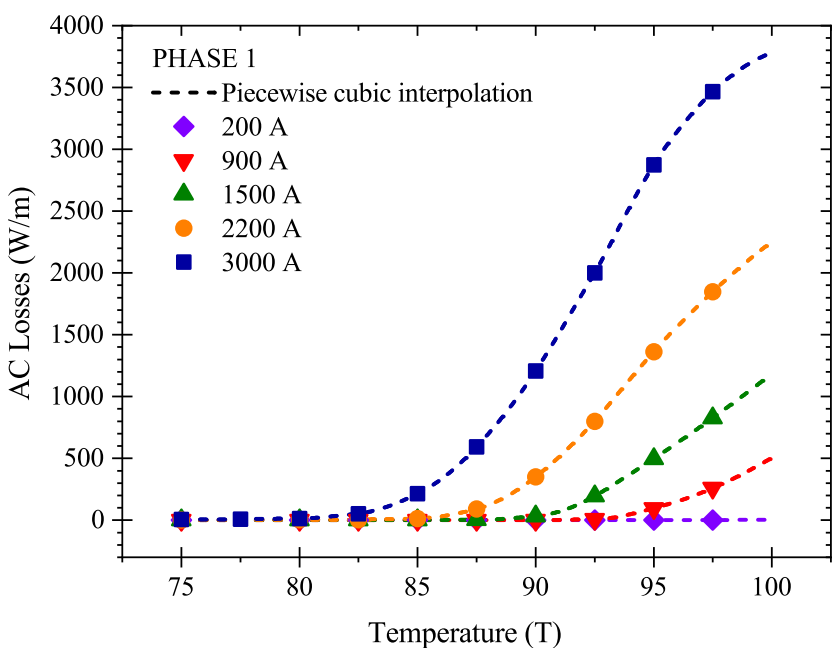

Fig. 9: The estimated losses for a number of transport RMS currents for phase one of the simulated cable, against temperature.

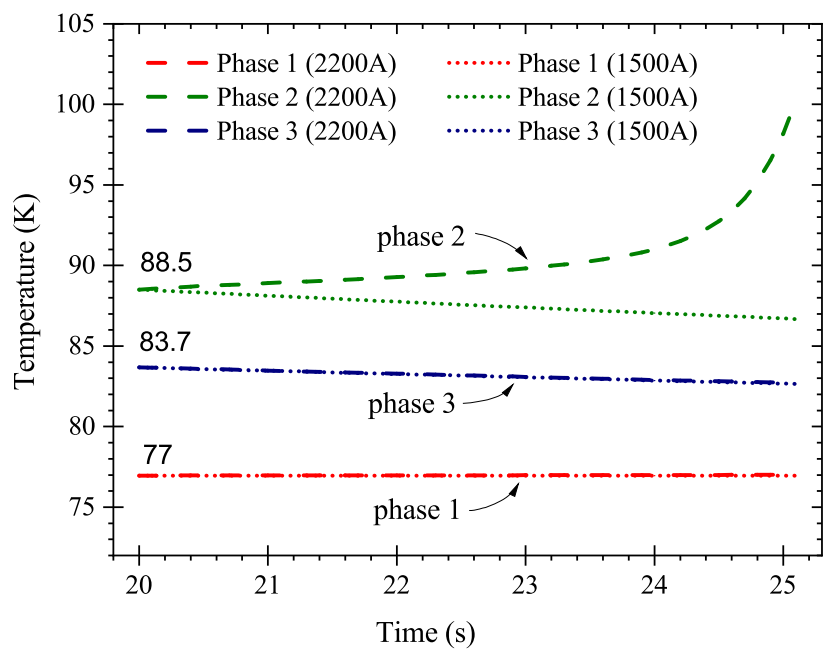

Fig. 10: A demonstration of how the second phase drives the re-quench. Current is applied at 20 seconds.

differs. In the cross-section model, each tape is defined and has finite dimensions, whereas in the axisymmetric model, the conducting material is approximated as a monoblock mixture of silver and HTS taking the shape of a hollow cylinder. As this hollow cylinder is a solid body, its cross-sectional area is larger than the area of individual tapes that, in reality, occupy that space. So, when a certain amount of heat (in watts) that is actually generated in the surface area of $A_{\mathrm{hts}} \mathrm{m}^{2}$, is applied on a larger area, for example $1.5 \times A_{\mathrm{hts}} \mathrm{m}^{2}$, the resulting maximum temperature would be lower in the domain with the larger area due to the larger thermal capacity.

However, if it is kept in mind that the actual temperature within each phase may be higher than the one predicted by the axisymmetric model, it is a reasonable approximation for the purposes of identifying the cable's recovery time. That is so due to the fact the amount of Joule heating is equal in both models and therefore the same amount of heat generated in

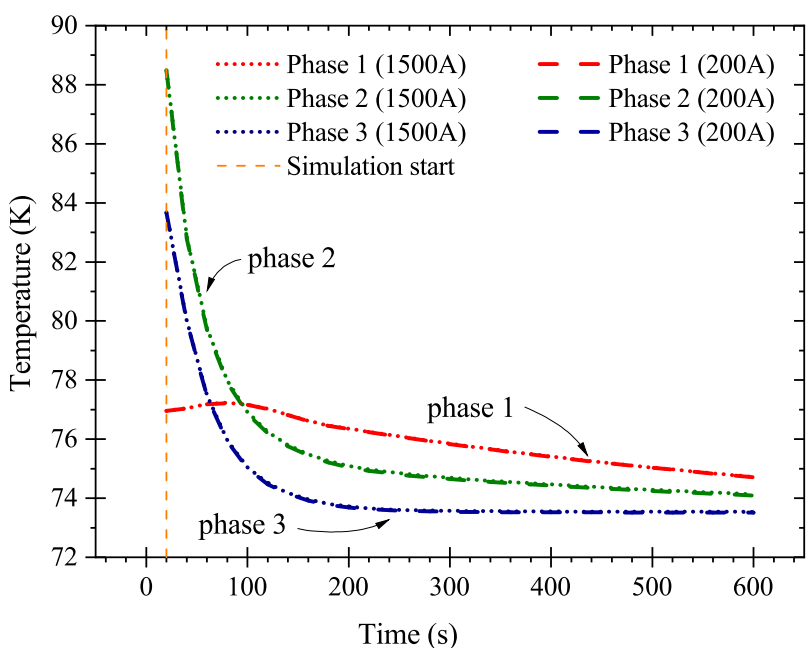

Fig. 11: The temperature of each phase for 600 seconds across several values for transport current. Current is applied at 20 seconds.

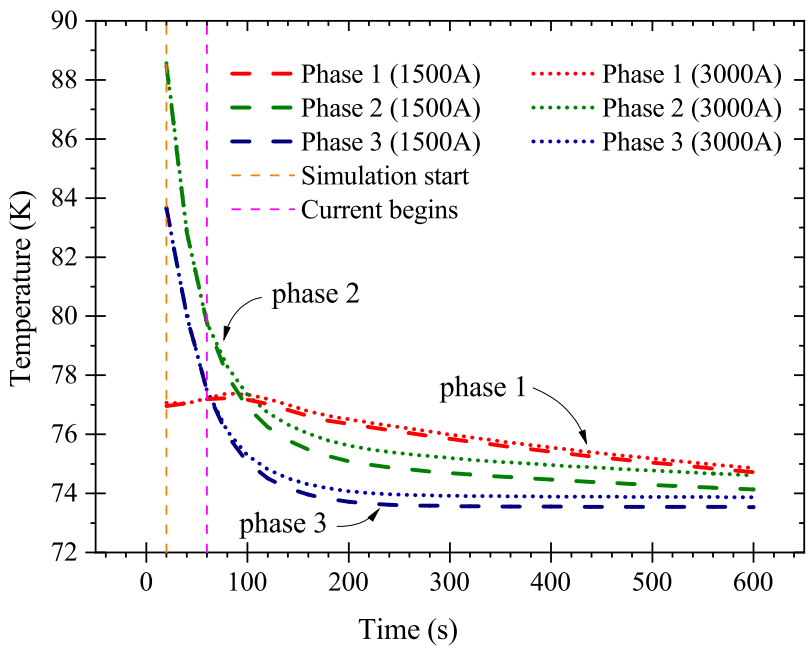

Fig. 12: The temperature of each phase for $600 \mathrm{~s}$ across several values for transport current. Current is applied at 60 seconds.

the cross-sectional model will eventually be transferred to the coolant.

On an office PC with Intel i7-6700 CPU, $3.40 \mathrm{GHz}$, with 16 GB of RAM, the simultaneous COMSOL simulation for the fault duration completed in 55 hours, compared to 15 hours for the parallel-solved parametric sweep on a cluster.

\section{Post-Fault Recovery Results}

The simulated versus literature [36] temperature of each phase after the fault has been cleared (after $t=0.1 \mathrm{~s}$ ) is shown on Fig. 7. Observing the obtained results (solid lines), the behaviour of the cooling of each phase is as expected. The first phase, being closest to the supply flow, has the highest rate of cooling. This is followed by the third phase. The second phase's position in the middle of the cable, sandwiched between the other phases and surrounding insulation, takes the longest to cool down. 
The same behaviour can be observed in the results obtained from [36] (dashed lines). However, the rate of cooling of all phases is visibly lower in the literature. This is particularly visible for the second phase which practically does not decrease in temperature between $t=0.5$ and $t=1$ seconds. Due to PPLP's low thermal conductivity (which would likely be higher along the length of the cable due to the multi-layered structure), it would be unlikely for the surrounding insulation to heat up and saturate to the value of the temperature of the second phase in less than one second. This is corroborated in the FEM model. This observation suggests one of two things, or perhaps both at the same time. The first possibility is there exists a difference between the finite element and finite difference model when it comes down to solving this problem, possibly in the way space is discretized (a 1D or a 2D domain). The second possibility is a difference in the material properties or certain calculations (for heat transfer coefficients for example).

[36] suggests all phases have returned to below their critical temperature $(100 \mathrm{~K})$ at about 20 seconds. The simulation, on the other hand, finds that happens at about 5 seconds and by the tenth second, each phase has comfortably recovered. It must be kept in mind that the temperatures in the 2Daxisymmetric subroutine are lower than the actual temperatures of the cable, as described at the end of Section II-E and so care must be taken when trying to find the recovery time of a cable design in this way.

Once the phases have returned to a temperature below critical, they may be subjected to current again. The magnitude of that current will dictate whether the cable will quench again or slowly return to steady state temperatures, and if it returns to steady state then how fast it will do so.

\section{A. Very little or no current during recovery}

If no current or current considerably smaller than the critical current (such as $I_{t}=283$ A peak or 200 A RMS) is applied, there will be a negligible amount of heat generated (Fig. 9). This is investigated in [36] and it is discovered the temperature of the cable's liquid nitrogen approaches steady state after around 5 hours, and has practically reached steady state after around 8 hours.

The results are illustrated in Fig. 8. The predicted temperature distributions via the coupled FEM model strongly resemble the results from literature. Both models agree - at no point does the coolant temperature exceed $77.5 \mathrm{~K}$. What they disagree on are the temperatures at the end of the cable (where supply and return flow join), and at the cable outlet. The simulated temperature decreases somewhat faster than the results from [36], with the difference being comparable to the difference in the steady state results. Notably, by hour 8, the disagreement has completely vanished.

\section{B. Large current during recovery}

An investigation is performed to see how the cable's operation will be affected during recovery if the applied current is non-negligible. Four RMS transport current values are selected: 900 A, 1500 A, 2200 A and 3000 A. These correspond to $0.29,0.48,0.70$ and 0.96 of phase one's 4400 A-peak critical current, respectively. The $2 \mathrm{D}$ cross-section subroutine is then used to estimate the losses in $\mathrm{W} / \mathrm{m}$ in each phase while under isothermal conditions for temperatures from $75 \mathrm{~K}$ to $97.5 \mathrm{~K}$ with a step of $2.5 \mathrm{~K}$. An interpolated function can be extracted from these results, for the purposes of obtaining the value of losses against temperature, for each phase for a given transport current. Such an approximation for the simulated cable from [23], [36] is displayed in Fig. 9, where the datapoints are transformed into a continious function through a piecewise cubic interpolation.

The computationally expensive simulation of the $H$ formulation for periods longer than several cycles can now be avoided; the remaining simulation can be entirely performed using only the heat transfer equation in the 2D cross-section subroutine. Only one cross-section needs to be simulated as it was shown the longitudinal change in temperature of each phase was insignificant in the FEM model - the crosssection at 600 metres was chosen as it is closest to the peak temperature from both literature and this work.

It is very important to note that if the axisymmetric subroutine is allowed to generate its own heat, the final results may inaccurately show a current may run without re-quenching the cable. This is discussed in the previous sections - it is because the axisymmetric model underestimates the maximum achievable temperature due to the difference between conductor areas.

Let current be applied at $t=20 \mathrm{~s} .900 \mathrm{~A}$ and $1500 \mathrm{~A}$ may run effectively without a noticeable change in temperature thus, in practice they do not affect the recovery of the cable (Fig. 11). However, at the next simulated value (2200 A), the heat generated becomes large enough to throw off the thermal balance and quench the superconductor. A closer look at the temperature rise shows that the rise is driven by the second (middle) phase, because it has the highest temperature. This can be seen in Fig. 10 - portrayed are the temperature recoveries during a $1500 \mathrm{~A}$ and $2200 \mathrm{~A}$. The heat generated in phase two of the 2200 A simulation increases the temperature steadily.

The actual upper limit of current that would not re-quench the superconductor is between $1500 \mathrm{~A}$ and $2200 \mathrm{~A}$, and its value may be determined more precisely if necessary.

Let current be applied at $t=60 \mathrm{~s}$. At that point in time, the temperature of each layer has dropped sufficiently and no re-quench occurs even with the highest value of 3000 A RMS. However, as illustrated in Fig. 12, the introduction of the 3000 A current increases the temperature in each layer such that the recovery takes longer than when a lower current value is applied.

\section{CONCLUSION}

This work presents the efficient finite element multiphysics modelling of a three-phase cable in the Triax configuration. The model verification confirms that proposed FEM model is viable and may be implemented robustly using existing commercial simulation tools (e.g. COMSOL). No external additions are necessary. Moreover, the proposed methodology 
implies that the entire simulation may be performed over a moderately reasonable amount of time using low computational resources when certain approximations are exploited namely, using the $H$-formulation only on a limited number of cross-sections along the cable. The simulations completed in less than 20 hours for a decoupled distributed parametric sweep of all cross-sections (on a computing cluster) and less than 60 hours for a fully coupled model on an office PC that simulated all cross-sections at the same time.

Certain assumptions have been used throughout all simulations. The only sources of heating are the three superconducting phases and copper neutral. This means no AC losses in the cable core or other metallic components are considered. In the 2D-axisymmetric model, all phases are assumed to be monoblocks with a width equal to the width of the tape and thermal conductivity equal to the thermal conductivity of HTS material in line with literature. All physical properties, except resistivity and critical current density, are assumed to be temperature-independent. Ohmic losses during the initial steady-state calculation are assumed negligible. The magnetic field dependence of the tape in this paper has been assumed to be similar to the field dependence of an earlier tape from literature.

Additionally, the results presented in this paper are tied to this specific cable design. While they may be indicative of the behaviour of such a cable in general, they should not be interpreted outside of the scope of this paper.

It was shown that for HTSCs the operators have to wait tens of seconds for a HTSC to be cooled, although the typical fault clearance time is a fraction of a second. Even HTSCs can be back into the operation after tens of seconds from the fault, the transport current has to be limited for several minutes to allow the cable to cool down further to normal operation temperatures.

\section{ACKNOWLEDGMENT}

Special thanks to Dr Wescley Tiago Batista de Sousa from Karlsruhe Institute of Technology for generously providing parameters from his research. This work was supported by the United Kingdom Engineering and Physical Sciences Research Council [grant number EP/N509747/1]. The authors acknowledge the use of the IRIDIS High Performance Computing Facility, and associated support services at the University of Southampton, in the completion of this work.

\section{REFERENCES}

[1] D. I. Doukas, "Superconducting transmission systems: Review, classification, and technology readiness assessment," IEEE Transactions on Applied Superconductivity, vol. 29, no. 5, pp. 1-5, 2019.

[2] N. Hu, M. Toda, A. N. Ozcivan, T. Yagai, M. Tsuda, and T. Hamajima, "Fault current analysis in a tri-axial HTS cable," IEEE Transactions on Applied Superconductivity, vol. 20, no. 3, pp. 1288-1291, 2010.

[3] N. Hu, M. Toda, T. Watanabe, M. Tsuda, and T. Hamajima, "Recovery time analysis in a tri-axial HTS cable after an over-current fault," Physica C: Superconductivity and its Applications, vol. 471, no. 21-22, pp. 12951299, 2011.

[4] N. Hu, K. Cao, D. Wang, M. Song, D. Miyagi, M. Tsuda, and T. Hamajima, "Transient thermal analysis of a tri-axial HTS cable on fault current condition," Physica C: Superconductivity, vol. 494, pp. 276-279, 2013.
[5] Z. Zhuonan, Z. Jiahui, L. Huifeng, Q. Ming, L. Zhenming, D. Kaizhong, and W. Yasen, "Magnetic-thermal coupling analysis of the cold dielectric high temperature superconducting cable," IEEE Transactions on Applied Superconductivity, vol. 23, no. 3, pp. 5400404-5400 404, 2013.

[6] H. Sun-Kyoung, K. Sung-Kyu, K. Jin-Geun, P. Minwon, Y. In-Keun, L. Sangjin, S. Kideok, and A. R. Kim, "Transient characteristic analysis of a tri-axial HTS power cable using PSCAD/EMTDC," IEEE Transactions on Applied Superconductivity, vol. 23, no. 3, pp. 5400104 $5400104,2013$.

[7] B. Shu, F. M. Wang, Z. Y. Liu, J. T. Li, Z. J. Zhao, J. H. Li, and Z. Liang, "Fault analysis of HTS power transmission cables," in 2013 IEEE International Conference on Applied Superconductivity and Electromagnetic Devices, 2013, Conference Proceedings, pp. 543-545.

[8] J. Li, Z. Zhao, B. Shu, X. Han, X. Ma, B. Bian, J. Li, and Z. Liang, "Fault analysis for $110 \mathrm{kV}$ HTS power cables," IEEE Transactions on Applied Superconductivity, vol. 24, no. 5, pp. 1-5, 2014.

[9] J. Fang, H. F. Li, J. H. Zhu, Z. N. Zhou, Y. X. Li, Z. Shen, D. L. Dong, T. Yu, Z. M. Li, and M. Qiu, "Numerical analysis of the stability of HTS power cable under fault current considering the gaps in the cable," Physica C: Superconductivity, vol. 494, pp. 319-323, 2013.

[10] Z. M. Li, W. Liu, J. H. Zhu, M. Qiu, X. D. Zheng, and J. Fang, “Temperature and current distribution of high temperature superconducting cable itself under large fault current," in 2015 IEEE International Conference on Applied Superconductivity and Electromagnetic Devices (ASEMD), 2015, Conference Proceedings, pp. 151-152.

[11] J. Zhu, H. Li, M. Qiu, J. Fang, X. Chen, and W. Yuan, "Magnetothermal coupling modeling for the stability analysis of a $110 \mathrm{kV} / 3 \mathrm{kA}$ high temperature superconducting cable under fault current conditions," Journal of Superconductivity and Novel Magnetism, vol. 28, no. 2, pp. 607-613, 2014.

[12] L. Yin, X. F. Ma, X. N. Li, K. Zhang, X. D. Zheng, J. Fang, J. H. Zhu, and M. Qiu, "Analysis of high temperature superconducting cable model under fault current," in 2015 IEEE International Conference on Applied Superconductivity and Electromagnetic Devices (ASEMD), 2015, Conference Proceedings, pp. 266-267.

[13] X. Wang, H. Ueda, A. Ishiyama, M. Yagi, S. Mukoyama, M. Ohya, T. Masuda, N. Kashima, S. Nagaya, and Y. Shiohara, "Over-current characteristics of a 20-m-long YBCO model cable," IEEE Transactions on Applied Superconductivity, vol. 19, no. 3, pp. 1722-1726, 2009.

[14] X. Wang, A. Ishiyama, M. Ohya, and N. Fujiwara, "Over-current characteristics of 66-kV RE123 HTS power cable," IEEE Transactions on Applied Superconductivity, vol. 21, no. 3, pp. 1013-1016, 2011.

[15] A. Ishiyama, X. Wang, H. Ueda, T. Uryu, M. Yagi, and N. Fujiwara, "Over-current characteristics of 275-kV class YBCO power cable," IEEE Transactions on Applied Superconductivity, vol. 21, no. 3, pp. 10171020, 2011.

[16] K. Adachi, K. Shiohara, H. Sugane, T. Kitamura, N. Mido, T. Hasegawa, M. Konno, and M. Iwakuma, "Sudden short-circuit test of $22 \mathrm{kV} \mathrm{YBCO}$ triaxial superconducting cable," IEEE Transactions on Applied Superconductivity, pp. 1-1, 2018.

[17] W. T. B. d. Sousa, D. Kottonau, J. Bock, and M. Noe, "Investigation of a concentric three-phase HTS cable connected to an SFCL device," IEEE Transactions on Applied Superconductivity, vol. 28, no. 4, pp. 1-5, 2018.

[18] A. Petrov, J. Pilgrim, and I. Golosnoy, "Revisiting the homogenized domain model for fast simulation of AC transport power losses in first generation high temperature superconducting tapes and cables," Physica C: Superconductivity and its Applications, vol. 557, pp. 33 - 40, 2019.

[19] A. N. Petrov, J. A. Pilgrim, and I. O. Golosnoy, "2D finite element modelling of the AC transport power loss in multi-layer Bi-2223 cables," Journal of Physics: Conference Series, vol. 1559, p. 012134, 2020.

[20] H. Jie, T. Yuejin, W. Bin, L. Jingdong, R. Li, S. Jing, K. Wu, L. Xiaoyu, $\mathrm{X}$. Ying, and W. Shu, "Thermal analysis of HTS power cable using 3-D FEM model," IEEE Transactions on Applied Superconductivity, vol. 23, no. 3, pp. $5402404-5402404,2013$.

[21] E. Shashi Menon, "Chapter five - fluid flow in pipes," in Transmission Pipeline Calculations and Simulations Manual, E. Shashi Menon, Ed. Boston: Gulf Professional Publishing, 2015, pp. 149-234. [Online]. Available: https://www.sciencedirect.com/science/article/pii/ B9781856178303000055

[22] M. Furuse, S. Fuchino, K. Agatsuma, T. Masuda, M. Ohya, S. Honjo, T. Mimura, and Y. Noguchi, "Stability analysis of HTS power cable with fault currents," IEEE Transactions on Applied Superconductivity, vol. 21, no. 3, pp. 1021-1024, 2011.

[23] E. Shabagin, C. Heidt, S. Strauß, and S. Grohmann, "Modelling of 3D temperature profiles and pressure drop in concentric three-phase HTS power cables," Cryogenics, vol. 81, pp. 24-32, 2017. 
[24] R. Brambilla, F. Grilli, and L. Martini, "Development of an edge-element model for AC loss computation of high-temperature superconductors," Superconductor Science and Technology, vol. 20, no. 1, pp. 16-24, 2007.

[25] Z. Hong, A. M. Campbell, and T. A. Coombs, "Numerical solution of critical state in superconductivity by finite element software," Superconductor Science and Technology, vol. 19, no. 12, pp. 1246-1252, 2006.

[26] R. Zermeno, V. M. Sørensen, M. P. Pedersen, N. F. Mijatovic, and A. B. Ambrahamsen, "Fast 2D simulation of superconductors: a multiscale approach," in Proceedings of the COMSOL Conference Milan. COMSOL, 2009.

[27] M. Zhang and T. A. Coombs, "3D modeling of high-Tc superconductors by finite element software," Superconductor Science and Technology, vol. 25 , no. 1 , p. $015009,2012$.

[28] V. M. R. Zermeno, F. Grilli, and F. Sirois, "A full 3D time-dependent electromagnetic model for Roebel cables," Superconductor Science and Technology, vol. 26, no. 5, p. 052001, 2013.

[29] B. Shen, F. Grilli, and T. Coombs, "Review of the AC loss computation for HTS using H formulation," Superconductor Science and Technology, vol. 33, no. 3, p. 033002, 2020.

[30] F. Grilli, E. Pardo, A. Stenvall, D. N. Nguyen, Y. Weijia, and F. Gomory, "Computation of losses in HTS under the action of varying magnetic fields and currents," IEEE Transactions on Applied Superconductivity, vol. 24, no. 1, pp. 78-110, 2014.

[31] F. Grilli, "Numerical modelling of high temperature sperconducting tapes and cables," Thesis, Swiss Federal Institute of Technology Lausanne, 2003.

[32] V. M. R. Zermeno, A. B. Abrahamsen, N. Mijatovic, B. B. Jensen, and M. P. Sørensen, "Calculation of alternating current losses in stacks and coils made of second generation high temperature superconducting tapes for large scale applications," Journal of Applied Physics, vol. 114, no. 17, p. 173901, 2013.

[33] Z.-F. Jiang, X.-F. Gou, and T.-M. Shen, "Numerical simulation of quench initiation and propagation in multi-filamentary $\mathrm{Bi} 2 \mathrm{Sr} 2 \mathrm{CaCu} 2 \mathrm{Ox}$ round wires at high magnetic fields," Journal of Applied Physics, vol. 125, no. 16, p. 163901, 2019.

[34] K. Berger, J. Leveque, D. Netter, B. Douine, and A. Rezzoug, "AC transport losses calculation in a Bi-2223 current lead using thermal coupling with an analytical formula," IEEE Transactions on Applied Superconductivity, vol. 15, no. 2, pp. 1508-1511, 2005.

[35] J. Duron, F. Grilli, B. Dutoit, and S. Stavrev, "Modelling the E-J relation of high-Tc superconductors in an arbitrary current range," Physica C: Superconductivity, vol. 401, no. 1-4, pp. 231-235, 2004.

[36] W. T. Batista de Sousa, D. Kottonau, and M. Noe, "Transient simulation and recovery time of a three-phase concentric HTS cable," IEEE Transactions on Applied Superconductivity, vol. 29, no. 5, pp. 1-5, 2019.

[37] S. Elschner, E. Demencik, B. Douine, F. Grilli, A. Kudymow, M. Stemmle, S. Strauss, V. Zermeno, and W. Goldacker, "New experimental method for investigating AC losses in concentric HTS power cables," IEEE Transactions on Applied Superconductivity, vol. 25, no. 3, pp. 1-5, 2015.

[38] M. Stemmle, F. Merschel, M. Noe, and A. Hobl, "AmpaCity - advanced superconducting medium voltage system for urban area power supply," in 2014 IEEE PES T\&D Conference and Exposition, 2014, Conference Proceedings, pp. 1-5.

[39] S. Choi, W. Nah, J. H. Kim, J. Joo, Y. H. Jo, and K. W. Ryu, "AC transport current loss of horizontally attached $\mathrm{Bi}-2223 / \mathrm{Ag}$ tapes," IEEE Transactions on Appiled Superconductivity, vol. 14, no. 2, pp. 1894 1897, 2004.

[40] COMSOL Multiphysics User's Guide, 5.2a, Comsol Inc., Burlington, USA.

[41] M. Kalsia and R. S. Dondapati, "Influence of flow rate and heat flux on the temperature distribution in long length counter flow cooled cold dielectric HTS cables," Physica C: Superconductivity and its Applications, vol. 567, p. 1353549, 2019. 